\title{
Perioperative Risk Assessment for Surgery of Consecutive Cases with Ventricular Septal Rupture: Seeking A Novel and Comprehensive Method
}

\author{
Lu Wang, BA, ${ }^{1}$ Ting Hai, MD,${ }^{1}$ Yi Feng, MD,${ }^{1}$ Qiaoyu Han, MD,${ }^{1}$ Yaru Li, BA, ${ }^{1}$ Yu Chen, MD,${ }^{2}$ \\ Wei Yang, MD, ${ }^{2}$ Zhou Zhao, MD, ${ }^{2}$ Luyang Jiang, $\mathrm{MD}^{1}$
}

${ }^{1}$ Department of Anesthesiology, Peking University People's Hospital, Beijing, China;

${ }^{2}$ Department of Cardiac Surgery, Peking University People's Hospital, Beijing, China

\section{ABSTRACT}

Background: Ventricular septal rupture (VSR) is a rare but lethal complication occurring after acute myocardial infarction. The aim of our study was to review the singlecenter experience of surgery for VSR and seek a comprehensive evaluation process for early mortality.

Methods: Patients undergoing surgical repair for postinfarction VSR in our institution retrospectively were evaluated from Jan. 2006 to Dec. 2019. The endpoint of the study was mortality within 30 days after VSR surgery, which was divided into survivors and nonsurvivors. The calibration and discrimination of two risk evaluation systems (European System for Cardiac Operative Risk Evaluation II (EuroSCORE II) and the Society of Thoracic Surgeons (STS) risk score) in total were compared by Hosmer-Lemeshow, and the area under the receiver operating characteristic curve (AUC). Risk factors in subsets were assessed by logistic regression analysis.

Results: Twenty-three patients undergoing surgery for VSR repair were reviewed, and the early mortality after surgery was $34.8 \%(N=8)$. The expected mortality predicted by EuroSCORE II was $24.3 \%$, and that of the STS score was $12.2 \%$. Both the EuroSCORE II and STS risk evaluation systems showed positive calibration in predicting mortality (H-L: $P=0.117$ and $P=0.346$, respectively) but poor discriminative power ( $\mathrm{AUC}=0.633$ and 0.575 ). Significant predictors determined by univariate analysis were concomitant coronary artery bypass grafting $(\mathrm{CABG})(P=0.035)$ and postoperative continuous renal replacement therapy (CRRT) $(P=0.008)$.

Conclusion: Early mortality of VSR after surgery remains high, and the evaluation process is complicated. The performances of the two risk evaluation systems were not optimal, but EuroSCORE II was more accurate than STS. Patients with lower preoperation EuroSCORE II, concomitant CABG during repair, and no need for CRRT after surgery may have a better early survival rate.

Received March 1, 2021; accepted March 22, 2021.

Correspondence: Luyang fiang, MD, No.11, Xi Zhi Men Nan Street, Xicheng District, Beijing, 100044, China, Telephone 13269054309 (e-mail: jiangluyang@pkuph.edu.cn).

\section{INTRODUCTION}

Ventricular septal rupture (VSR) is a rare but catastrophic complication of acute myocardial infarction (MI). The incidence of VSR is approximately $0.2 \%$ of all patients after $\mathrm{MI}$ in the thrombolytic therapy era [Crenshaw 2000]. The mortality of VSR is notably high, being more than $90 \%$ with conservative treatment, while after surgical intervention, the mortality of VSR varies from $30 \%$ to $50 \%$ in different centers [Loisance 1991; Singh 2017; Marek 2018; Anders 2005; Arnaoutakis 2012]. Different risk evaluation systems have been utilized to predict mortality after cardiac surgery, and at present, two widely accepted systems are the European System for Cardiac Operative Risk Evaluation II (EuroSCORE II) and the Society of Thoracic Surgeons (STS) risk score. Accurate preoperative risk evaluation systems are critical for measuring the magnitude of risks after surgery, choosing treatment projects, providing consultation for patients, and improving quality programs [Carla 2012].

The mortality of patients is determined not only by some preoperative variables included in the risk evaluation systems but also by other intra- or postoperative variables. Our study attempts to identify a more precise assessment system preoperatively and characterize other predictors such that we could have optimal perioperative management and thorough assessment of VSR repair patients.

\section{MATERIALS AND METHODS}

This study was retrospective. From January 2006 to December 2019, patients diagnosed with postinfarction VSR undergoing surgical repair at Peking University People's Hospital were included in the study, and patients' clinical records were extracted from the Haitai database. Inclusion criteria were isolated VSR repair or VSR repair combined with other cardiac surgeries. Diagnosed criteria were definite MI (history of extreme chest pain, changes on electrocardiogram, and level of myocardial enzyme in plasma), systolic murmur over the precordium, transthoracic echocardiography (TTE) detecting dropout of ventricular septum and left-to-right shunt across the ventricle with color Doppler. The expected mortality of each patient was calculated separately through two risk evaluation systems by the official algorithms online: EuroSCORE II can be accessed at http://www.euroscore.org/ 
calc.html, and the STS risk evaluation system can be accessed at http://riskcalc.sts.org/STSWebRiskCalc273/de.aspx. The calibration and discrimination were described as the predictive efficacy for mortality yielded by the two systems.

We defined the outcome endpoint as mortality within 30 days after surgery, regardless of cause. The patients were divided into nonsurvivors and survivors groups, and the expected mortality of the two groups was compared by each system to make an accurate comparison between the two systems. Data were collected on the following variables not included in two risk evaluation systems: location of VSR, perforation size, time interval from MI to VSR, time interval from VSR to surgery, peak brain natriuretic peptide (BNP) and cardiac troponin I (cTnI) before surgery, blood product infusion during surgery, aortic cross-clamping time and assist time during cardiopulmonary bypass (CPB), concomitant coronary artery bypass grafting (CABG) or valve repair surgery, level of lactic acid in blood gas and dosage of inotrope score [Wernovsky 1995] (dopamine $\mu \mathrm{g} . \mathrm{kg}^{-1}$. min-

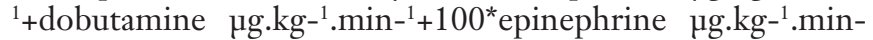
${ }^{1}+100^{*}$ norepinephrine $\mu \mathrm{g} \cdot \mathrm{kg}^{-1} \cdot \mathrm{min}^{-1}+15^{*}$ milrinone $\mu \mathrm{g} \cdot \mathrm{kg}^{-1}$. min- ${ }^{1}$ ) before leaving the operation room, length of ventilator-depend, length of intensive care unit, postoperative residual, postoperative continuous renal replacement therapy (CRRT), left ventricular ejection fraction (LVEF) ratio (post/ pre), and time interval from surgery to death or discharge.

Continuous variables are presented as the mean \pm standard deviation or median, while categorical variables are presented as percentages. Discrimination of the evaluation system, which could discriminate between nonsurvivors and survivors, is assessed by the area under the receiver operating characteristic curve (AUC), and the power of discrimination is considered excellent if AUC is $>0.8$ and acceptable if AUC is $>0.7$. Calibration of the evaluation model, which could measure differences between observed and expected results, is assessed by the Hosmer-Lemeshow (H-L) goodness-of-fit statistic, and $P>0.05$ is considered well calibrated. Univariate logistic regression analysis of risk factors for mortality is performed to obtain odds ratios (ORs) and 95\% confidence intervals (CIs), and a $P$ value $<0.05$ is considered to be significant. All statistical analyses are performed with SPSS 22.0.

\section{RESULTS}

The records of 23 patients who underwent VSR repair, from January 2006 to December 2019, in our center were reviewed and analyzed. Demographics were collected, according to factors included in the two evaluation systems. Some baseline parameters of all patients are presented in Table 1. (Table 1)

Among the 23 patients who underwent VSR repair, eight died within 30 days after surgery (six died of low cardiac output, one died of central neutral system complications after extubation, and one died of acute artery embolism: thrombosis from the superior mesenteric artery and renal artery). The overall observed mortality rate was $34.8 \%$, the expected mortality predicted by EuroSCORE II was $24.3 \%$, and expected mortality predicted by the STS score was $12.2 \%$; therefore, EuroSCORE II was closer to the actual mortality than the STS score. Both the EuroSCORE II and STS risk evaluation systems showed positive calibration in predicting mortality (H-L: $P=0.117$ and $P=0.346$, respectively) but underestimated the observed mortality. Both EuroSCORE II (AUC $=0.633<0.7)$ and STS score $(\mathrm{AUC}=0.575<0.7)($ Table 2$)$ showed poor discriminative power for all patients. (Table 2)

The overall patients were divided into nonsurvivor and survivor groups ( 8 vs. 15) after surgery. Significant risk factors for early mortality by univariate logistic regression analysis were body mass index (BMI) $(P=0.021)$, concomitant CABG $(P=0.035)$, and postoperative CRRT $(P=0.008)$. Patients with lower BMI, with concomitant CABG and not treated with CRRT, were more likely to survive within 30 days (Table 3). (Table 3) The expected mortality of the two groups was calculated and compared by EuroSCORE II (23.9 vs. 12.3, $P=0.98)$ and the STS risk evaluation system (8.8 vs. $4.2, P$ $=0.76$ ). There was no significance between the two groups, according to the two systems, which is equal to the poor discrimination obtained by the AUC of the two systems.

\section{DISCUSSION}

The mean time for the development of VSR after acute MI was reported to be one day in the thrombolytic era [Crenshaw 2000]. Among 5745 patients after percutaneous coronary intervention in ST-elevation MI from the Assessment of Pexelizumab in Acute Myocardial Infarction, VSR occurred at a median of 7.7 hours after symptom onset [French 2010]. Advanced age, female sex, chronic kidney disease, and congestive heart failure are independent risk factors for postinfarction VSR [Jones 2014]. From 1990 to 2007, the mortality for acute MI complicated by VSR, due to sudden and severe hemodynamic deterioration, remained high and was unchanged with advances in pharmacology and improved surgical techniques, but surgical repair was associated with a survival benefit for in-hospital mortality compared with conservative treatment [Moreyra 2010]; therefore, definitive surgical VSR repair remains a useful choice for treatment.

Precise preoperative risk evaluation models are important to decisions regarding anesthetic and surgical strategies in cardiac practice. EuroSCORE was developed from a European database of 19030 patients with cardiac surgeries from 1995 to 1999 to evaluate the degree of risk after surgery [Nashef 1999]. Initially, EuroSCORE was well accepted but showed some limitations in assessing mortality with the advent of surgical techniques; therefore, it was revised and updated as EuroSCORE II in 2012. Although some evidence demonstrated that EuroSCORE II underestimated mortality risk compared with the STS risk score model [Gul 2013], its feasible characteristic with fewer variables and application in various cardiac procedures caused it to be widely used. Compared with EuroSCORE II, the STS risk score periodically has been revised and updated since being established, and the added variables had a significant influence on mortality after cardiac practice [Shih 2015], which contributed to the 
superiority of the STS risk score to EuroSCORE II for evaluating risk magnitude and some other outcomes of surgery, such as renal failure and length of stay. In addition, the STS risk model predicts mortality, according to different surgical types, which could improve the accuracy of a specific model but limit it to isolated CABG, isolated valve surgery or CABG

Table 1. Baseline clinical characteristics of patients

\begin{tabular}{|c|c|}
\hline Characteristics & Description \\
\hline Age (years) & $63.8 \pm 11.3$ \\
\hline Female & $11(47.8)$ \\
\hline BMI $\left(\mathrm{kg} / \mathrm{m}^{2}\right)$ & $24.4 \pm 3.8$ \\
\hline $\operatorname{Scr}(\mu \mathrm{mol} / \mathrm{L})$ & $105.6 \pm 56.4$ \\
\hline Diabetes & $7(30.4)$ \\
\hline Hypertension & $14(60.9)$ \\
\hline Cerebral vascular disease & $2(8.7)$ \\
\hline Peripheral vascular disease & $2(8.7)$ \\
\hline Poor morbidity & $2(8.7)$ \\
\hline Atrial flutter and fibrillation & $1(4.3)$ \\
\hline Tobacco use & $10(43.5)$ \\
\hline Alcohol use & $6(26.1)$ \\
\hline Previous cardiac interventions & $4(17.4)$ \\
\hline MI within 90 days & $20(87)$ \\
\hline \multicolumn{2}{|l|}{ Pulmonary hypertension } \\
\hline No & $9(39.1)$ \\
\hline Moderate $31-55 \mathrm{mmHg}$ & $8(34.8)$ \\
\hline Severe $>55 \mathrm{mmHg}$ & $6(26.1)$ \\
\hline \multicolumn{2}{|l|}{ NYHA } \\
\hline 1 & $0(0)$ \\
\hline ॥ & $1(4.3)$ \\
\hline III & $11(47.8)$ \\
\hline IV & $11(47.8)$ \\
\hline \multicolumn{2}{|l|}{ LVEF } \\
\hline$>50 \%$ & $12(52.2)$ \\
\hline $31 \%-50 \%$ & $10(43.5)$ \\
\hline $21 \%-30 \%$ & $1(4.3)$ \\
\hline Preoperative IABP & $15(65.2)$ \\
\hline CPR before surgery & $2(8.7)$ \\
\hline Perioperative ECMO & $2(8.7)$ \\
\hline Elective surgery & $9(39.1)$ \\
\hline Concomitant other surgery & $18(78.3)$ \\
\hline
\end{tabular}

BMI, body mass index; $C A B G$, coronary artery bypass grafting; CPR, cardiopulmonary resuscitation; ECMO, extracorporeal membrane oxygenation; IABP, intra-aortic balloon pump; LVEF, left ventricular ejection fraction; MI, myocardial infarction; NYHA, New York Heart Association; Scr, serum creatinine combined with valve surgery [Shahian 2009; O'Brien 2009; Shahian 2009]. All risk evaluation systems have benefits and drawbacks. Previous studies only compared these evaluation systems to predict mortality after CABG or valve surgeries [Lingtong 2018; Ad 2016], while evidence on risk models for VSR repair is scarce, and a better system remains to be identified.

In our study, the mortality within 30 days after repair was $34.8 \%$, which was consistent with previously reported data [Singh 2017; Marek 2018]. The EuroSCORE II and STS risk scores showed positive calibration but underestimated the actual mortality, which can be explained by two aspects. Repair for VSR is complex and challenging. Ischemic and necrotic myocardial tissues initially are fragile, making them difficult to repair, and mortality in the first 24 hours of presentation was highest compared with seven days and more than seven days $(>60 \%, 54.1 \%, 18,4 \%$ respectively) [Arnaoutakis 2012; Jones 2014]. Therefore, the time of surgery is highly important, and an effective therapy is to delay the surgery and enable the stability of the fragile tissue to improve. However, many patients may suddenly experience circulation collapse in a state of cardiogenic shock in the initial period of presentation such that they have to be under emergency surgery by CPB in the operating room. The complexity of surgery and critical state of patients cause VSR repair to have a relatively high mortality among all types of cardiac surgery [Jones 2014]. The EuroSCORE II and STS risk score are established to evaluate normal cardiac practices, such as CABG and valve replacements; therefore, it is reasonable that the two risk models underestimated the observed mortality.

In addition, a patient's clinical situation cannot be completely evaluated precisely with limited risk variables. The variables included in the two risk models occupy only part of the risk factors for VSR repair mortality, and certain important factors are ignored. The predictors for mortality with surgical intervention include age, female sex, higher serum creatinine levels, lack of hypertension or lung disease [Arnaoutakis 2012], time from MI to VSR, time from VSR to surgery, residual VSR [Simon 2009], posterior rupture, early repair [Anders 2005], diabetes, time from MI to surgery, lack of a preoperative intra-aortic balloon pump, elevated preoperative right atrial pressure [Blanche 1992], right ventricular (RV) function, preoperative and evolution of the clinical status, and type of repair [Labrousse 2002], according to different studies. EuroSCORE II and STS risk score both include risk

Table 2. Predicted efficacy of the two evaluation systems

\begin{tabular}{lcc}
\hline & EuroSCORE II & STS risk score \\
\hline Expected mortality (\%) & 24.3 & 12.2 \\
AUC & 0.633 & 0.575 \\
H-L statistics & 0.117 & 0.346 \\
\hline
\end{tabular}

AUC, area under receiver operating characteristic curve; $\mathrm{H}-\mathrm{L}$ statistics, Hosmer-Lemeshow statistics 
Table 3. Univariate analysis of risk factors for early mortality

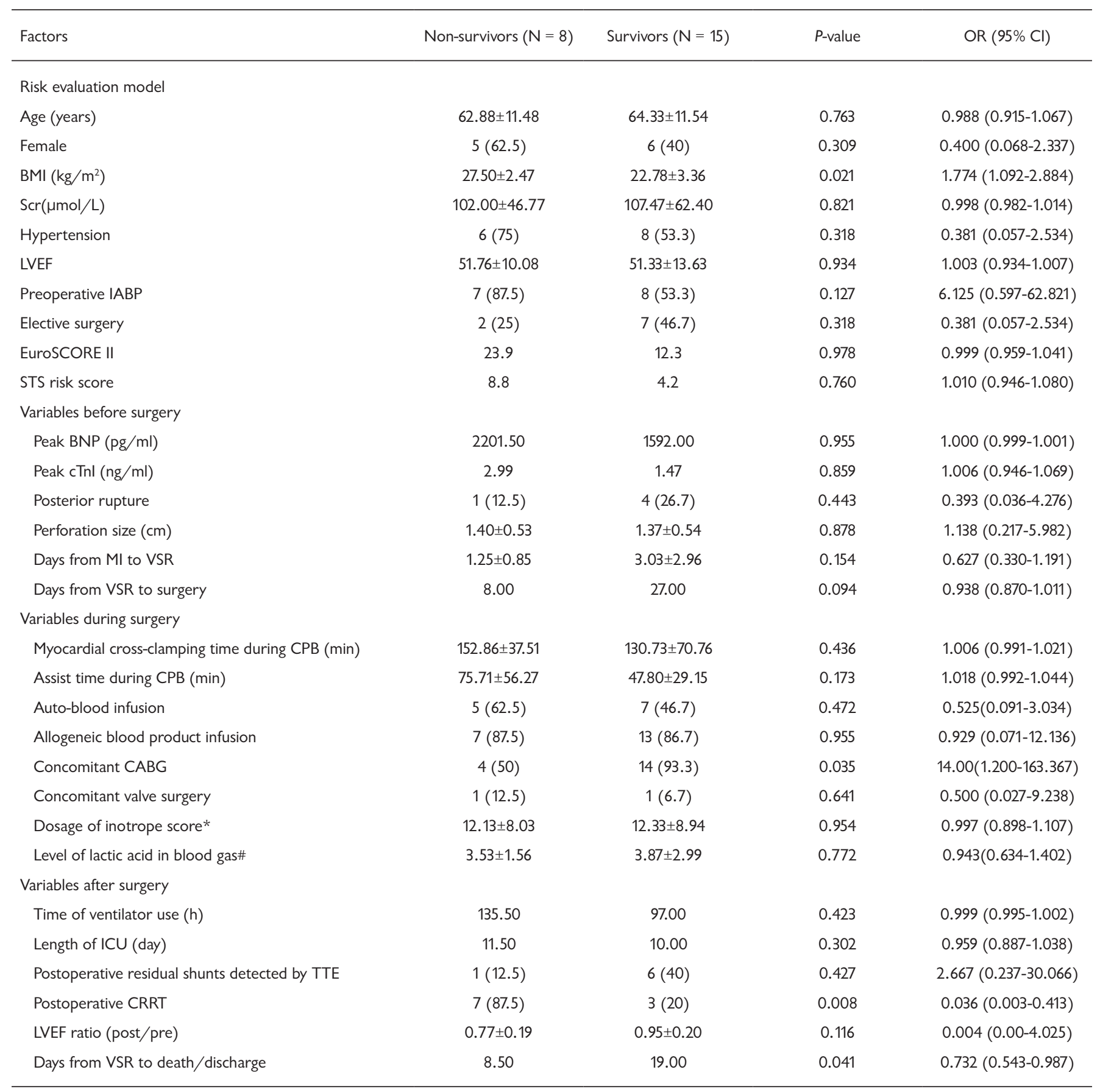

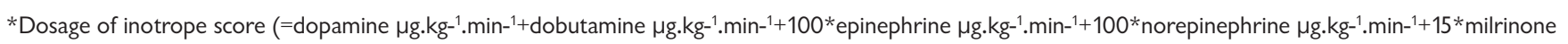
$\left.\mu \mathrm{g} \cdot \mathrm{kg}^{-1} \cdot \mathrm{min}^{-1}\right)$ intravenous when transferred to ICU after surgery

\#Level of lactic acid in blood gas before leaving the operation room

BMI, body mass index; BNP, brain natriuretic peptide; Cl, confidence interval; CPB, cardiopulmonary bypass; CRRT, continuous renal replacement therapy; cTnl, cardiac troponin I; ICU, intensive care unit; LVEF, left ventricular ejection fraction; MI, myocardial infarction; OR, odds ratio; RV, right ventricle; Scr, serum creatinine; TTE, transthoracic echocardiography; VSR, ventricular septal rupture 
factors, such as age, sex, and serum creatinine, and STS risk score involves some other factors, such as hypertension and detailed time of MI. However, both evaluation systems overlook some surgical factors for VSR, such as time from VSR to surgery, anatomic location of rupture and RV dysfunction. These factors are associated with the effect of surgery, and the ignored factors may lead to underestimation of mortality. The EuroSCORE II is closer to the observed mortality than the STS risk score, possibly because the STS risk score may include too many irrelevant variables with VSR repair that influence the integral accuracy of the risk model.

We included some factors that may be associated with mortality but were not mentioned in the two systems. Concomitant CABG is protective in our center, but its role in survival is controversial among different studies. Philip and colleagues concluded that concomitant CABG did not have an effect on early or late survival [Pang 2013], and some existing evidence supported our findings [Takahashi 2015; Barker 2003]. We advise VSR patients routinely to have coronary angiography before surgery and perform $\mathrm{CABG}$, if possible, to improve early outcomes. Low cardiac output after surgery is a leading cause of death in our center $(75 \%)$, can occur from acute ventricular dysfunction, myocardial ischemia, and prolonged aortic cross-clamp and is a main reason for acute kidney injury (AKI) and the use of CRRT [Chew 2019]. Postoperative use of CRRT may be a negative indicator for AKI and low cardiac output, and we should be aware of serum creatinine levels after surgery. Maintaining organ perfusion, correcting acidosis and undertaking some other prompt maneuvers improve patient outcomes.

In addition to detailed evaluation, taking some measures to improve the stability of hemodynamics is critical for anesthesiologists during surgery. Induction for VSR repair is challenging because of contradictions between general anesthesia drugs and the stimulation of intubation, and it is of vital importance to maintain the stability of hemodynamics during induction. VSR can lead to a left-to-right shunt, and $\mathrm{RV}$ volume overload, pulmonary blood flow and left ventricle volume overload eventually increase. The size of the rupture, level of pulmonary vascular resistance (PVR) and systemic vascular resistance (SVR) and their ratio, and left and right ventricular function can decide the extent of shunt [Birnbaum 2002]. RV volume overload is a reversible factor associated with RV dysfunction, a strong predictor for survivors, in contrast to left ventricular dysfunction, RV infarction or ischemia [Labrousse 2002]. To diminish the magnitude of the shunt, one important goal is to reduce left ventricular afterload. Efforts should be made to avoid hypocapnea and excessive hyperoxemia, which could decrease the ratio of PVR and SVR, maintain normocapnia and minimize oxygen supply, if possible [Ryoji 2007].

Extracorporeal membrane oxygenation (ECMO) before surgical repair of a postinfarction VSR may serve as a rescue strategy, stabilizing the hemodynamic status and internal environment, to provide a bridge to have surgery opportunity [Matos 2020]. In our center, two patients had ECMO but failed to survive, due to low cardiac output. Interventional therapy, such as transcatheter closure of postmyocardial infarction VSR, is an innovation for patients. The present study showed that mortality within 30 days after interventional therapy was 23\% [Assenza 2013], which is still high. In addition, the interventional procedure is difficult because of fragile and necrotic tissues but with residual shunts. However, transcatheter closure of VSR provides an opportunity to decrease the shunt for later surgical repair. ECMO, in addition to interventional treatment, may be a better solution, and more high-volume studies are needed to confirm the effect.

This study has some limitations. First, the sample size may be too small because the study is a single-center study, and the low incidence of VSR could affect the accuracy of our results. Second, our study is retrospective, and biases in data collection are inevitable. Surgical techniques have advanced in recent decades, which may have reduced the mortality of surgery. In addition, the risk evaluation model could show the best performance, when it is applied to situations similar to conditions of its origin, such as race, region, and applications. The STS risk score has its own preferred surgeries, and EuroSCORE II may be suitable for all cardiac surgeries in a center, not for specific procedures. However, our study is exploring a better risk evaluation system for VSR and provides a reasonable interpretation of the results. Further multicenter studies are needed to investigate the risk evaluation model and risk predictors for VSR surgical repair.

The mortality of postinfarction VSR surgical repair is notably high, and patients with lower mortality by EuroSCORE II before surgery, concomitant CABG during repair and no need for CRRT after surgery may have better early outcomes. It is important to make a full evaluation to achieve optimal anesthetic and perioperative management for VSR patients. However, risk evaluation is a merely referable process, and clinicians should use their own judgment and discretion for various clinical situations.

\section{REFERENCES}

Ad N, Holmes SD, Patel J, Pritchard G, Shuman DJ, Halpin L. 2016. Comparison of EuroSCORE II, Original EuroSCORE, and The Society of Thoracic Surgeons Risk Score in Cardiac Surgery Patients. Ann Thorac Surg 102

Anders J, Hans L, Per J, Marianne H, Kjell R. 2005. Surgical repair of post infarction ventricular septal defects: a national experience. Eur J Cardiothorac Surg. 27.

Arnaoutakis GJ, Zhao Y, George TJ, Sciortino CM, McCarthy PM, Conte JV. 2012. Surgical repair of ventricular septal defect after myocardial infarction: outcomes from the Society of Thoracic Surgeons National Database. Ann Thorac Surg. 94:436-43; discussion 443-4.

Assenza GE, McElhinney DB, Valente AM, et al. 2013. Transcatheter closure of post-myocardial infarction ventricular septal rupture. Circ Cardiovasc Interv. 6:59-67.

Barker TA, Ramnarine IR, Woo EB, et al. 2003. Repair of post-infarct ventricular septal defect with or without coronary artery bypass grafting in the northwest of England: a 5-year multi-institutional experience. Eur J Cardiothorac Surg. 24.

Birnbaum Y, Fishbein MC, Blanche C, Siegel RJ. 2002. Ventricular septal rupture after acute myocardial infarction. $\mathrm{N}$ Engl J Med. 347:1426-32. 
Blanche C, Khan SS, Matloff JM, et al. 1992. Results of early repair of ventricular septal defect after an acute myocardial infarction. J Thorac Cardiovasc Surg. 104.

Carla P, de Villiers Jonker I, Lezelle B, Smit Francis E. 2012. Cardiac surgery risk-stratification models. Cardiovasc J Afr. 23.

Chew STH, Hwang NC. 2019. Acute Kidney Injury After Cardiac Surgery: A Narrative Review of the Literature. J Cardiothorac Vasc Anesth. 33.

Crenshaw BS, Granger CB, Birnbaum Y, et al. 2000. Risk factors, angiographic

patterns, and outcomes in patients with ventricular septal defect complicating acute myocardial infarction. GUSTO-I (Global Utilization of Streptokinase and TPA for Occluded Coronary Arteries) Trial Investigators. Circulation. 101:27-32.

French John K, Hellkamp Anne S, Armstrong Paul W, et al. 2010. Mechanical complications after percutaneous coronary intervention in ST-elevation myocardial infarction (from APEX-AMI). Am J Cardiol. 105.

Gul KA, Murat K, Mete H, et al. 2013. Comparison of original EuroSCORE, EuroSCORE II and STS risk models in a Turkish cardiac surgical cohort. Interact Cardiovasc Thorac Surg. 16.

Jones BM, Kapadia SR, Smedira NG, et al. 2014. Ventricular septal rupture complicating acute myocardial infarction: a contemporary review. Eur Heart J. 35:2060-8.

Labrousse L, Choukroun E, Chevalier JM, et al. 2002. Surgery for post infarction ventricular septal defect (VSD): risk factors for hospital death and long-term results. Eur J Cardiothorac Surg. 21.

Lingtong S, Wen G, Yiwei P, et al. 2018. Assessment of three risk evaluation systems for patients aged $\geq 70$ in East China: performance of SinoSCORE, EuroSCORE II and the STS risk evaluation system. PeerJ. 6.

Loisance DY, Lordez JM, Deleuze PH, Dubois-Rande JL, Lellouche D, Cachera JP. 1991. Acute postinfarction septal rupture: long-term results. Ann Thorac Surg. 52.

Marek P, Jan H, Nedal O, Zdenek T, Jana S, Jan V. 2018. Surgical treatment of postinfarction ventricular septal defect: risk factors and outcome analysis. Interact Cardiovasc Thorac Surg. 26.

Matos D, Madeira M, Nolasco T, Neves JP. 2020. The role of extracorporeal membrane oxygenation in an acute basal ventricular septal rupture. Eur J Cardiothorac Surg. 57:799-800.

Moreyra AE, Huang MS, Wilson AC, Deng Y, Cosgrove NM, Kostis JB. 2010. Trends in incidence and mortality rates of ventricular septal rupture during acute myocardial infarction. Am J Cardiol. 106:1095-100.

Nashef SA, Roques F, Michel P, Gauducheau E, Lemeshow S, Salamon R. 1999. European system for cardiac operative risk evaluation (EuroSCORE). Eur J Cardiothorac Surg. 16.

O'Brien SM, Shahian DM, Filardo G, et al. 2009. The Society of Thoracic Surgeons 2008 Cardiac Surgery Risk Models: Part 2-Isolated Valve Surgery. Ann Thorac Surg. 88.

Pang PY, Sin YK, Lim CH, et al. 2013. Outcome and survival analysis of surgical repair of post-infarction ventricular septal rupture. J Cardiothorac Surg. 8:44.

Ryoji I, Keizo K, Shigeru S, Setsuro O. 2007. Anesthesia for repair of ventricular septal rupture after acute myocardial infarction. J Clin Anesth. 19.

Shahian DM, O'Brien SM, Filardo G, et al. 2009. The Society of Thoracic Surgeons 2008 Cardiac Surgery Risk Models: Part 1-Coronary Artery Bypass Grafting Surgery. Ann Thorac Surg. 88.

Shahian DM, O'Brien SM, Filardo G, et al. 2009. The Society of Thoracic Surgeons 2008 Cardiac Surgery Risk Models: Part 3-Valve Plus Coronary Artery Bypass Grafting Surgery. Ann Thorac Surg. 88.

Shih T, Paone G, Theurer PF, McDonald D, Shahian DM, Prager RL. 2015. The Society of Thoracic Surgeons Adult Cardiac Surgery Database Version 2.73: More Is Better. Ann Thorac Surg. 100.

Simon M, Reda I, Arsène-Joseph B, et al. 2009. Postinfarction ventricular septal defects: towards a new treatment algorithm. Ann Thorac Surg. 87.

Singh V, Rodriguez AP, Bhatt P, et al. 2017. Ventricular Septal Defect Complicating ST-Elevation Myocardial Infarctions: A Call for Action. Am J Med. 130: 863.e1-863.e12.

Takahashi H, Arif R, Almashhoor A, Ruhparwar A, Karck M, Kallenbach K. 2015. Long-term results after surgical treatment of postinfarction ventricular septal rupture. Eur J Cardiothorac Surg. 47:720-4.

Wernovsky G, Wypij D, Jonas RA, et al. 1995. Postoperative course and hemodynamic profile after the arterial switch operation in neonates and infants. A comparison of low-flow cardiopulmonary bypass and circulatory arrest. Circulation 92:2226-35. 